\title{
Dynamical approaches to modelling developmental gene regulatory networks
}

\author{
Nicholas Geard $^{a, *}$ and Kai Willadsen ${ }^{b}$ \\ ${ }^{a}$ School of Electronics and Computer Science \\ University of Southampton \\ Southampton, SO17 1BJ, United Kingdom \\ Tel: +44 (0)23 80594470 \\ Fax: +44 (0)23 80599179 \\ nlg@ecs.soton.ac.uk \\ ${ }^{b}$ Frankfurt Institute for Advanced Studies \\ Johann Wolfgang Goethe University \\ Ruth-Moufang-Str. 1, 60438 Frankfurt am Main, Germany \\ Tel: +49 (0)6979847616 \\ Fax: +49 (0)6979847611 \\ willadsen@fias. uni-frankfurt.de \\ ${ }^{*}$ Corresponding Author
}

This is a preprint of an article accepted for publication in Birth Defects Research C: Embryo Today. Copyright (C)2009 Wiley-Liss, Inc., A Wiley Company. 


\begin{abstract}
The network of interacting regulatory signals within a cell comprises one of the most complex and powerful computational systems in biology. Gene regulatory networks play a key role in transforming the information encoded in a genome into morphological form. To achieve this feat, gene regulatory networks must respond to and integrate environmental signals with their internal dynamics in a robust and coordinated fashion. The highly dynamic nature of this process lends itself to interpretation and analysis in the language of dynamical models. Modelling provides a means of systematically untangling the complicated structure of gene regulatory networks, a framework within which to simulate the behaviour of reconstructed systems and, in some cases, suites of analytic tools for exploring that behaviour and its implications. This review provides a general background to the idea of treating a regulatory network as a dynamical system, and describes a variety of different approaches that have been taken to the dynamical modelling of gene regulatory networks.
\end{abstract}

\title{
Introduction
}

Gene regulatory networks (GRNs) encode the patterns of interacting signals responsible for the up and down regulation of genes. GRNs integrate internal and external signals to ensure that a cell exhibits a response appropriate to its current environmental context. As such, GRNs are an important locus of developmental control: while epigenetic and environmental factors play an important role, GRNs act throughout development to ensure that the correct types of cell are produced in the correct place and at the correct time (Davidson, 2001; Carroll et al., 2001).

During development, the potential of each cell is progressively restricted toward some terminally differentiated type. A cell's fate is strongly correlated with the pattern of genes that it expresses. A wide variety of signals, both from within a cell and from without, can influence the activation of a gene. Many of these signals are proteins that are themselves the result of gene transcription. The activation of each gene can be influenced by multiple signals, and each signal can play multiple regulatory roles. The topology of the resulting regulatory system is therefore not a simple, linear pathway, but a complex network of interacting genes and signals. The conditions under which a gene becomes active are specified in the regulatory sequence encoded in the genome. Understanding the relationship between GRNs and development is necessary in order to address the question of how aspects of development are linked, via the structure and dynamics of GRNs, back to the heritable information encoded in an individual's genome (Levine and Davidson, 2005).

The amount of data available for the study of GRNs is greater than ever before. However, far from this being the end of the story, technologies such as genome sequencing have made us 
aware of the scale of the problem confronting us. Cataloguing the protein-coding regions in the genome represents only the first step in understanding how complex interactions between genes and biochemical molecules control the developmental process (Quackenbush, 2007). Understanding the structure and dynamics of the interaction networks in which these components are embedded will be necessary before a comprehensive understanding of development is possible. In this endeavour, modelling will be a crucial tool (Kitano, 2002).

An understanding of GRNs also offers the promise of insight into disease. Many diseases are a result of changes (e.g., induced by environmental factors) to the function of GRNs, particularly to those that operate during development (Chan and Bonini, 2000; Olson, 2006; Edwards and Myers, 2007). One remarkable property of GRNs is their robustness to perturbation. However, models have revealed that this stability is not be localised to any specific component within the GRN, but is rather an emergent property of the complex dynamics across the network (Kitano, 2004; Schadt and Lum, 2006). Understanding how developmental GRNs work can help to explain the ways and circumstances in which they fail, as well as how to target effective interventions.

This review aims to provide an overview of the wide variety of approaches to modelling the dynamic behaviour of GRNs.

\section{Modelling aims and approaches}

\section{Modelling aims}

Models are simplified representations of real systems. In exchange for sacrificing some fidelity, a model can provide new insight into the system, either by filtering out non-essential detail such that structural and behavioural patterns can be discerned, or by allowing manipulation and exploration of a kind not possible with the real system.

The complexity of biological systems means that a major challenge in modelling is deciding on an appropriate level of detail to include. Too much detail may result in a complicated model with reduced explanatory power, in which the essential nature of the process of interest is obscured. On the other hand, too little detail risks omitting critical processes and mechanisms, resulting in a model whose behaviour is not an accurate representation of the real system. Deciding how much detail to include in a model will be determined by the resources available (i.e., data, methodological tools) and by the question that motivates the model. We identify four classes of motivation - integration of empirical data, identification and characterisation of structural, dynamic or functional modules, development of theoretical insights and generation of testable hypotheses - although clearly any given model may overlap across classes.

First, models allow researchers to integrate empirical data into a meaningful theoreti- 
cal framework. As gene expression data becomes ever-more readily available, information management tools become correspondingly more important for analysis and communication. Standardised protocols and platforms such as the systems biology markup language (Hucka et al., 2003), Cytoscape (Shannon et al., 2003) and BioTapestry (Longabaugh et al., 2008) enable data and models to be easily shared between researchers. For example, Swiers et al. (2006) used published data to create a model of the GRN underlying the specification of the hematopoietic stem cell that has enabled new insights into the effects of perturbation, as well as acting as a focal point for the organisation and integration of future data.

Models also enable the identification of repeated patterns or modules in a system. These patterns, which may occur in the structure or dynamics of a system, can be thought of as fundamental building blocks that are assembled in various ways to produce more complex structures and behaviours (Savageau, 2001; Alon, 2007; Davidson and Levine, 2008). Approaching GRNs in terms of the meso-level components from which they are constructed provides a means of dealing with their otherwise intractable complexity. Examples of modules with important dynamic consequences include positive feedback circuits, which enable a transient signal to induce a stable cellular response (Xiong and Ferrell, 2003), and double-negative feedback circuits, which play a role in the selection and stabilisation of terminal cell fates (Johnston et al., 2005).

The use of mathematical and computational formalisms to construct GRN models has enabled researchers to relate biological systems to general dynamical theory. In so doing, a range of powerful theoretical and analytical tools become available to investigate the behaviour of these systems (Thomas, 1998; Tyson et al., 2001). An outcome of this approach is that the key components, interactions and variables that determine a system's behaviour can be identified, offering insight into possible targets for intervention (Schadt and Lum, 2006).

Finally, model building also plays a valuable role in the experimental cycle, as an aid to hypothesis formation and the interpretation of empirical data (Kitano, 2001). The complex and nonlinear structure of biological systems, combined with the varying timescales on which different biological processes act, makes it particularly difficult to develop intuitions about how regulatory systems operate. Building a formal model of such a system requires all assumptions about the timing and connectivity of regulatory elements to be made explicit. Modelling can therefore provide a valuable check on intuitions during the development of hypotheses (McAdams and Shapiro, 1995).

\section{Modelling approaches}

Along with the many different motivations for modelling, there are a wide variety of approaches. Any GRN model can be broken into several components: a useful distinction is between parts 
lists, topology, control logic and dynamics (Schlitt and Brazma, 2007). Any given model may focus on one or more of these components while de-emphasising the others; however, in the construction of dynamical models - the focus of this review - at least some consideration must be given to the constituent elements of a GRN and how they interact.

Parts lists: A parts list is a collection and description of the constituent elements that make up a GRN, such as genes and their products, the regulatory sequences to which regulatory factors bind, and other species that play a role in regulation. The parts list by itself can provide a perspective for comparing species, but is more commonly the starting point for the development of a more informative model. The arrival of high-throughput experimental techniques has resulted in a surge of data for the development of this type of model; for example, putative binding sites can be identified via sequence analysis (Osada et al., 2004).

Topology: Topological models describe how the parts of a system interact, often viewed as a network in which nodes represent parts and edges represent interactions between those parts. Both nodes and edges may have different meanings depending on context: for example, in a GRN a (directed) edge between two genes $A$ and $B$ may indicate that the product of gene $A$ binds to the regulatory region of gene $B$, causing it to be up or down regulated (see Figure 1). In a protein-protein interaction network, an (undirected) edge between $A$ and $B$ typically indicates that the two proteins are capable of binding together. These networks can themselves be the subject of analysis (e.g., using statistical or graph theoretic approaches such as in Albert, 2005) or they can form the basis for dynamic models.

Control logic: The next stage, beyond identifying the existence of an interaction between two system components, involves discovering what rules govern that interaction. Many genetic interactions can be approximated by a logical function, the simplest example being that gene $B$ is expressed only in the presence of product $A^{\prime}$. Alternatively, gene $B$ may be expressed in the presence of product $A^{\prime}$, but only if product $C^{\prime}$ is not present. In other cases, interactions are more complex and quantitative accounts of protein concentrations and activation thresholds are necessary.

Dynamics: Once all components of a system and their interactions have been identified, numerical or computational simulation can be applied to explore how the system behaves over time. There is a wide variety of approaches to modelling system dynamics. While some smaller models are tractable to formal analytic techniques, even systems with a modest number of non-linear interactions between their elements can defy analytic approaches. As complexity is a defining feature of many dynamic GRN models, numerical or computational simulation is 
required in many cases.

We further distinguish three complementary approaches to the design of a dynamical model: 'reverse engineering', phenomenological approaches and statistical ensembles.

The reverse engineering of existing biological systems seeks to better understand their dynamics and function via the development of high fidelity models of (typically) small systems from available empirical knowledge. Each component in these models frequently corresponds to a particular element of the biological system under investigation. Numerical and computer simulations can then be used to make predictions about systems that are too complex to allow for analytical treatment (Hasty et al., 2001).

The phenomenological approach uses generalised models to reproduce observed biological behaviours, such as hysteresis or pattern formation. In these models, there may no longer be a direct mapping between components in the model and components in the biological system, however the high level behaviour of the system is preserved. Such models can be used to gain insight into, for example, the level of regulatory complexity required to produce a particular class of dynamic behaviour (Salazar-Ciudad et al., 2000).

A final approach comprises models that investigate the general principles of dynamical systems. Rather than seeking to understand individual systems, this approach seeks to characterise the behaviour of classes of GRNs with particular structural and dynamic properties. These approaches frequently use simplified descriptions of gene activation that allow much larger and more complex networks to be simulated than would otherwise be possible. A common technique is to generate a large number of random networks (an ensemble) governed by a specified set of local rules and observe the statistical properties of their global behaviour (Kauffman, 2004a,b).

Each of these approaches are necessary and complementary: local models of specific systems can be used to generate hypotheses that are directly testable by real experiments, as well as providing the impetus for developing more general theories; conversely, the study of general principles provides tools for the analysis of reverse-engineered systems; and abstract models can act as informed null hypotheses for real systems, providing a context within which to understand each system's unique characteristics.

\section{Gene networks as dynamical systems}

While different approaches to modelling GRNs focus on different levels of description-giving primacy to components or topology, for instance - regulatory networks are fundamentally dynamic entities, their functionality exposed through changing patterns of gene expression. As such, regulatory networks may be modelled within the framework of dynamical systems the- 
ory. Modelling a regulatory network as a dynamical system can provide insights into the link between structural and functional views of a system.

A variety of different dynamical systems modelling approaches have been used to simulate the behaviour of GRNs, and all of these models share some similarities in their approach and some common abstractions. All dynamical systems models focus on describing and simulating: (a) the state of the system, and (b) changes to the system state. In the context of a GRN, system state may represent the concentration levels of known regulatory factors, in which case changes to the state would represent concentration changes due to transcription, translation or decay events. While a system's state can be represented in many different ways, the ability to comprehensively describe a system's state at a given point in time, and to describe how successive temporal states are linked, are both key aspects of a dynamical systems formalism.

In the general parlance of dynamical systems theory, these elements give us states, transitions - changes from one state to another-and state spaces. A system's state space can be thought of as the collection of all of the possible states of the system, along with all possible transitions between states. While state spaces are an abstract construct, they provide a framework for thinking about the dynamics of a system. The most notable features of a state space are attractors and basins of attraction. An attractor of a system is a state (or set of states) towards which the system tends over time, whereas a basin of attraction is the set of states that tend towards a particular attractor; each attractor has an associated basin of attraction, and most systems of interest will have multiple attractors. In a real GRN, an attractor corresponds to the steady states (or stable oscillations) of a system, while basins of attraction correspond to the set of initial system states that will converge to a particular steady state.

Steady states of a regulatory system are interesting both because they are more easily experimentally observable, and because of the functional interpretations assigned to them. One early interpretation of steady states in a GRN model was that these states correspond to cell types (Kauffman, 1969). An updated interpretation is that steady states correspond more generally with alternate cell fates (Huang, 2004), in which different 'fates' may correspond to different functional regimes between which a cell can switch as required by environmental conditions. In support of this interpretation, recent work has uncovered the existence of attractor-like behaviour in cell dynamics, as recovered from gene expression time series data (Huang et al., 2005, 2007). The analogy between attractors and cell fates also provides a framework for drawing correspondences between the dynamics of a system and its function.

If attractors represent cell fates, then basins of attraction represent the initial condition leading to different cell fates. While some perturbations may move a system to another state in the same basin of attraction, others will push the system into a different basin, potentially one with a very different long term behaviour. Thus abnormal developmental trajectories, such as known mutations or defects, may be interpreted as perturbations to a wild-type initial state 
that result in initial conditions in a different basin of attraction. Dynamical systems models, and in particular simplified models such as Boolean networks, lend themselves to systematic exploration of the space of possible initial conditions of a system. If a model can accurately reproduce a system's behaviour, then it is in principle simple to test any initial condition or perturbation. In this way, it is possible to simulate different initial conditions, perturbations, or response to stimuli; for well-understood biological systems with known abnormal behaviour patterns, these cases may be used to assess potential predictive power of a dynamic model. An example of such a system is the Drosophila melanogaster segment polarity network, which is a well-characterised system that has been the subject of several modelling studies (von Dassow et al., 2000; Albert and Othmer, 2003; Chaves et al., 2005). For this system, not only was the wild-type behaviour of the network reproduced, but several known mutant conditions were also replicated and the existence of further difficult-to-observe steady states was hypothesised (Albert and Othmer, 2003).

\section{Models of gene regulatory networks}

The design and construction of a GRN model involves a variety of decisions: system states and variables can be logical or continuous; state transitions can be deterministic or stochastic; system elements can be updated all at once (synchronously) or independently (asynchronously); and spatial structure can be incorporated or excluded. This section reviews a range of different modelling approaches.

\section{Boolean networks and logical models}

Modelling regulatory networks as a set of logical elements has a long history. Perhaps the most well-known model, the Random Boolean Network (RBN) model, was originally designed as a highly abstract model of gene regulation to investigate whether certain observed characteris-

tics of genetic systems (e.g., developmental robustness) could be explained by network-level structures with highly idealised dynamics (Kauffman, 1969, 1993). The combination of simplicity, analytic tractability and generality of the model gave it broad appeal, and resulted in much theoretical work (e.g., Derrida and Pomeau, 1986; Luque and Solé, 2000; Socolar and Kauffman, 2003; Samuelsson and Troein, 2003) and several biologically-motivated extensions (e.g., Aldana, 2003; Harris et al., 2002).

In the RBN model, node (gene) activation is assumed to be Boolean - a node is always simply active or inactive, with no intermediate states - and regulation is a logical function of current node activities. Each node is influenced by some subset of the other nodes in the network, and these influences take the form of a logical function that determines what the 
next state of the node will be, given the current state of its influencing nodes (see Figure 2).

In the most general formulation, the network structure in RBNs is randomly generated, and the logical functions of nodes in the network are randomly selected. This purely random nature is what makes the RBN model a good basis for comparison with more biologically plausible networks: in order to understand what behaviours of a specific network are unusual, a set of baseline behaviours and properties are required to make a comparison. A significant contribution of the RBN model was the realisation that ordered behaviour could be obtained 'for free'; that is, without being specifically engineered into a system (Kauffman, 1993).

RBNs have several interesting properties, and make some broad general predictions about behaviour in regulatory systems. The model demonstrates three distinct behavioural regimes: stable (or ordered), critical and chaotic (or disordered), where the 'critical' regime is best described as the phase transition point between the stable and chaotic regimes. These regimes denote several related characteristics, including the robustness of attractors (Kauffman, 1993; Aldana, 2003), the fragmentation of state space (Willadsen et al., 2008), and the scaling of number of attractors (Socolar and Kauffman, 2003; Samuelsson and Troein, 2003). It has been proposed that regulatory systems operate within (or near) the critical regime - sometimes referred to as the 'edge of chaos' - as this regime is considered to be flexible without being uncontrollable or unstable (Kauffman, 1993, 2004b). Central to the importance of the critical regime is the existence of attractors that are highly robust both to initial conditions and to perturbation; results from the RBN model originally inspired the attractors-cell type analogy discussed above (Kauffman, 1969). In this context, the properties of the critical regime can be interpreted to provide baseline expectations about robustness, scaling of number of cell-fates with network size, and other system characteristics (Kauffman, 1993).

Studies of dynamic regimes have used the network structure of real regulatory systems, as reconstructed from experimental evidence, to create simplified RBN-based models of the largescale behaviour of these systems. The observed dynamics are consistent with the hypothesis that these systems operate near the critical regime (Balleza et al., 2008), and are unlikely to operate in the chaotic regime (Shmulevich et al., 2005). It is worth noting that while early studies of RBNs made much of the existence of the stable periodic attractor behaviour that is characteristic of the critical regime, the majority of published models of regulatory systems show dynamics involving only steady state attractors (Albert and Othmer, 2003; Li et al., 2004; Mendoza and Alvarez-Buylla, 1998); more plausibly, it is features such as the capacity for multistationarity (Kim et al., 2008) that make the critical regime significant. The robustness demonstrated by Boolean models of real regulatory systems (e.g., Albert and Othmer, 2003; Li et al., 2004) also supports the idea that regulatory systems are most likely to exist in a stable or critical regime.

Recent studies building on the theoretical background provided by RBNs include models of 
the cell-cycle networks in yeasts Saccharomyces cerevisiae (Li et al., 2004) and Schizosaccharomyces pombe (Davidich and Bornholdt, 2008a), the segment polarity network in Drosophila melanogaster (Albert and Othmer, 2003), and floral organ cell-type specification in Arabidopsis thaliana (Mendoza and Alvarez-Buylla, 1998; Chaos et al., 2006), as well as several theoretical analyses of these models (e.g., Chaves et al., 2006; Braunewell and Bornholdt, 2007; Irons and Monk, 2007; Willadsen and Wiles, 2007). The success of these studies provides some validation for the initially highly-abstract approach taken by the RBN model. In particular, modelling using a Boolean network approach indicates that important network dynamics may be reproduced with only network structure and simple regulatory information (Albert and Othmer, 2003); for at least some biological systems fine-tuning of kinetic parameters appears to be unnecessary.

\section{Scale-free and canalised Boolean networks}

The RBN model provides one possible baseline for comparison with real genetic regulatory systems. It also provides a theoretical framework in which different null hypotheses can be formulated. Two notable modifications to the RBN that provide alternative null hypotheses for regulatory network behaviour are scale-free Boolean networks and canalised Boolean networks.

Scale-free network models (and closely-related small-world networks) were proposed as better models for the structure of natural networks (Watts and Strogatz, 1998; Barabási and Albert, 1999). Scale-free network structures are prevalent in cellular systems, where they exhibit several important properties: short path length, which enables rapid communication between disparate parts of the network; and robustness to network perturbations (Albert and Barabási, 2002; Albert, 2005).

Studies of RBNs with a scale-free structure have found that the modified systems produce highly robust network behaviour that retains the interesting characteristics of the standard RBN model, while using a more biologically plausible network structure (Aldana, 2003). Stable and critical regime behaviours occurred for a wider range of network parametrisations; in other words, network behaviour was less sensitive to structural parameters like the connectivity of individual nodes (Aldana, 2003). The system also showed robustness characteristics typical of scale-free networks, such as exceptionally high robustness to random perturbation along with vulnerability to targeted attack (Albert et al., 2000). Finally, scale-free network structures appear to demonstrate better trade-offs between robustness and evolvability of the network (Aldana et al., 2007).

In contrast to scale-free variants, which alter only network structure, canalising Boolean network models alter the selection of Boolean functions that determine a system's dynamic behaviour (Kauffman, 1971; Harris et al., 2002). A canalising Boolean function is one in which 
one of the inputs to a node has the ability to override all other inputs for at least one value. For example, if gene $B$, when active, forces gene $A$ to be inactivated irrespective of the state of gene $A$ 's other inputs, then gene $A$ is said to be canalised by gene $B$.

Canalising functions are noteworthy for two reasons: they are more representative of the logical functions implemented by real regulatory networks (Harris et al., 2002); and they improve the robustness of network behaviour, again increasing the parameter range over which non-chaotic behaviour is observed (Kauffman et al., 2004). Specifically, it has been suggested that for Boolean network models of observed gene regulatory systems, using canalising Boolean functions produces behaviour that is stable while remaining close to criticality (Kauffman et al., 2003; Balleza et al., 2008); in contrast, a purely random selection of functions in otherwise similar networks is more likely to result in instability.

\section{Logic networks}

Generalised logical network models are a more descriptive relative of RBNs that aim to provide a standard method for describing regulatory interactions (Thomas, 1973) using either Boolean or multi-valued logic (Thomas and Kaufman, 1995). These networks are distinguished from simpler Boolean network models primarily by multi-value logic, asynchronous continuous-time dynamics and time-delay effects. Generalised logic networks provide a framework for modelling systems with multiple threshold-dependent effects (rather than the single threshold afforded by Boolean models) or for which timing effects are important.

Generalised logical networks have been used to study regulatory system behaviour in abstract terms (e.g., Mestl et al., 1995; Edwards and Glass, 2000), and also to model specific systems. Examples of biological systems models constructed using this paradigm include phage$\lambda$ (Thieffry and Thomas, 1995) and flower morphogenesis in Arabidopsis thaliana (EspinosaSoto et al., 2004).

\section{Differential equation models}

The Boolean approximation makes the assumption all genes are saturated either 'on' or 'off'. However, gene expression levels and product concentrations are continuous, rather than binary and genes may have different regulatory effects at different levels of expression. An alternative approach to modelling GRNs in situations where more precise concentration levels may be important is to use differential equations (DEs).

In a DE model, a system's state is described by a list of continuous variables representing gene expression levels. The transitions between states are defined in terms of update functions describing how gene expression levels change over time (see Figure 3). These functions can describe linear control, where the expression of a gene at time $t+\delta t$ depends linearly on a 
weighted sum of expression levels at time $t$ (Chen et al., 1999; D'haeseleer et al., 1999). More plausibly however, the interactions between components in real systems are nonlinear. In a nonlinear DE model, the weighted sum is modified by some transfer function, often sigmoidal in shape (Weaver et al., 1999). The resulting models can bear many similarities to connectionist models of neural systems (Hertz et al., 1991; Mjolsness et al., 1991; Wahde and Hertz, 2001; Vohradský, 2001).

DE models have several advantages over logical models. In principle, their more detailed representation of regulatory interactions provides a more accurate representation of the physical system under investigation. Additionally, there is a large body of dynamical systems theory that can be used to analyse such models (Strogatz, 1994). For example, bifurcation analysis provides tools for determining the critical values of parameters at which the behaviour of a system undergoes a qualitative change (see Figure 3 (b)). As with logical models, analysing DE models in terms of their dynamical properties can reveal how switches, oscillators and more complex behaviours are produced from network-level features such as interacting positive and negative feedback loops (Tyson et al., 2001, 2003; Angeli et al., 2004).

Compared to logical approaches, a disadvantage of DE models is that they contain a large number of kinetic parameters, while the number of systems for which detailed parameter values are known is very small, mostly restricted to very simple organisms such as phage$\lambda$ (Shea and Ackers, 1985). One approach to dealing with unknown parameter values is to use numerical analysis or computational learning techniques to fit the models. This approach has been successfully adopted in models of cell cycle control in Xenopus (Novak and Tyson, 1993) and the segment polarity network in Drosophila (von Dassow et al., 2000). In both cases, the models resulted in the formation of hypotheses about kinetic parameters or interactions that were later experimentally verified (von Dassow and Odell, 2002; Tyson et al., 2002). A further discovery resulting from this approach was that the dynamical behaviour of the segment polarity network was remarkably robust to variations in the parameter values (von Dassow et al., 2000). Similarly robust behaviour was observed for the signalling network containing the Notch-Delta pathway involved in Drosophila neurogenesis (Meir et al., 2002).

Despite being more detailed than logical models, DE models also make certain idealised assumptions about the systems they are studying. One simplifying assumption made by many DE models is that the relationship between rates of gene transcription and concentrations of active gene products is a linear one. However, the gene expression process is known to be highly complex, and regulation may exist at many stages of the expression process, including chemical and structural modification of DNA, gene transcription, post-transcriptional modification, transport and degradation of mRNA, translation and post-translational modification (Orphanides and Reinberg, 2002). Not only may each of these stages be regulated independently, but the time delays inherent in these processes may have significant effects on 
system dynamics, such as transforming steady states into oscillations (Mahaffy and Pao, 1984; Mahaffy, 1988; Smolen et al., 1999; Monk, 2003). One approach to relaxing this assumption is to explicitly incorporate such mechanisms in a model, for example, by including additional terms corresponding to protein degradation, or by modelling transcription and translation as discrete processes in which the production of messenger RNA depends upon the concentrations of protein transcription factors and the production of proteins depends on the concentrations of messenger RNAs (Vohradský, 2001; Goutsias and Kim, 2004). An alternative approach is to disregard the precise details of these intermediate processes but to introduce time delays accounting for the time they require (Zhu et al., 2007).

A second assumption of many DE models is that genes are expressed and proteins produced at a continuous rate. Again, this assumption does not always hold: in systems where the number of molecules involved is very small, the production and movement of individual molecules may be important. One possibility for modelling such systems is to use stochastic approaches.

\section{Stochasticity}

An implicit assumption made by many modelling approaches is that variation in product concentrations is smooth and control decisions are deterministic. In reality, the biochemical reactions in a GRN are subject to noise from both intrinsic and extrinsic sources. Low concentrations of regulatory molecules in a cell can cause reaction rates to fluctuate, and the products of gene transcription appear not continuously but in probabilistic bursts, leading to intrinsic noise (McAdams and Arkin, 1997; Thattai and van Oudenaarden, 2001). Extrinsic noise arises from the stochastic behaviour of other molecular species in a GRN's cellular context (Swain et al., 2002). In general, genes are activated when the concentration of signal molecules crosses a threshold. If the time taken for a concentration to reach its critical threshold varies, individual cells in a population may take different branches of a regulatory pathway and exhibit different behaviours. In some instances GRNs are buffered against this variation, and are capable of translating noisy inputs into ordered output (von Dassow et al., 2000). In other cases, this variation seems to be exploited by an organism to, for example, maintain an adaptive immune response, or ensure an appropriately diverse complement of sensory receptors (Rao et al., 2002; Losick and Desplan, 2008).

Several different stochastic modelling approaches have been proposed using both logical and DE formalisms. In the domain of logical models, one criticism of the standard RBN model is based on its use of deterministic synchronous updating (i.e., all nodes are always updated at each time step) which can be considered unrealistic. Relaxing this assumption introduces a level of indeterminism that, without other modifications, prevents the appearance of oscillating 
attractors (Klemm and Bornholdt, 2005). However, asynchronous variants of the RBN model have since been introduced (Di Paolo, 2001; Rohlfshagen and Di Paolo, 2004; Klemm and Bornholdt, 2005), and used to model the yeast cell cycle (Braunewell and Bornholdt, 2007; Davidich and Bornholdt, 2008b).

In the domain of DE models, one approach is to use stochastic differential equations, which extend the standard differential equation description of reaction dynamics to include a noise term (Rao et al., 2002). This equation can then be reformulated into a version describing the time evolution of a system in terms of a probability density function, or it can be simulated numerically using Monte Carlo methods. A characteristic of this approach is that concentrations are treated as continuous variables, which may not be appropriate if the number of signal molecules is very small (Rao et al., 2002). The stochastic simulation algorithm addresses this concern. Rather than considering rates of reaction, it focuses on individual reaction events, assigning each event a probability of occurring in a particular time slice (Gillespie, $1977,2001)$. While mathematically simple, the resulting equations are typically too large to be feasible solved, therefore numerical simulations are repeated many times in order to estimate the probable behaviour of the system. This formulation has been applied to modelling transcription in yeast (Blake et al., 2003; Raser and O'Shea, 2004), the mammalian circadian clock (Forger and Peskin, 2005) and the dynamics of differentiation to competence in Bacillus subtilis (Süel et al., 2007).

Both stochastic differential equations and the stochastic simulation algorithm are very computationally intensive, due to the requirement for multiple runs in order to estimate aggregate behaviour. One suggested method for increasing efficiency is to replace complex multi-step processes with time delays (Zhu et al., 2007). Another recently proposed technique to circumvent the multiple-run requirement uses pairwise comparisons to estimate covariance between the stochastic fluxes affecting reactants, resulting in equations solvable by standard numerical techniques (Goutsias, 2007).

\section{Hybrid models}

The hybrid approach to modelling recognises that real networks are characterised by a mixture of both discrete and continuous elements; for example, whereas a transition in cell behaviour, such as the onset of mitosis, is largely discrete, the concentration of a gene product may vary in a continuous fashion. In hybrid models, such dichotomies are addressed by incorporating elements of both the logical and continuous approaches to modelling. Again, the choice of which elements to implement as discrete versus continuous will be influenced by the target question and available data. However, the introduction of discrete values can simplify the control logic of a model, and potentially also reduce computational cost. The phage- $\lambda$ model of McAdams 
and Shapiro (1995) and single sea urchin gene model of Yuh et al. (1998) are examples of early models incorporating both discrete and continuous elements that were inspired by the control logic of electrical circuits. The latter model has since been extended to encompass the network of over forty genes involved in endomesoderm specification in the sea urchin embryo, making it one of the most completely characterised GRNs to date (Davidson et al., 2002; Ben-Tabou de-Leon and Davidson, 2007; Oliveri et al., 2008).

Several general frameworks for hybrid modelling of GRNs have also been proposed. Such approaches typically retain a continuous representation of time and product concentration, while expressing control logic in a discrete fashion. General hybrid modelling formalisms include the finite state linear model (Brazma and Schlitt, 2003), piecewise deterministic Markov processes (Kouretas et al., 2006), and piecewise-linear models (de Jong et al., 2004b), which have been applied to modelling the initiation of sporulation in Bacillus subtilis (de Jong et al., 2004a).

\section{Multicellularity and space}

A special consideration for models of developmental systems is the requirement for a multicellular perspective (Bolouri and Davidson, 2002). Although each individual cell contains the same genome - and hence the same gene network - the state of gene activation may vary between cells as localised signals cause diverging dynamic trajectories. Also, while many signals will operate intracellularly (i.e., upon other parts of the gene network in the same cell in which they are created), others may operate intercellularly (i.e., on parts of the gene network in surrounding cells). Thus, not only must the dynamical behaviour of any given cell be regulated, but it must also be coordinated with the behaviour of its neighbours.

One issue that must be addressed in multicellular models is the manner in which GRNs in neighbouring cells influence one another. One set of mechanisms requires physical contact between cells, with signals mediated by molecules bound to the cell membrane, or coupling via gap junctions. In GRN terms this can be represented by cross-regulatory interactions, in which the products produced in one cell are defined to influence receptors in neighbouring cells (e.g., von Dassow et al., 2000; Smith et al., 2007; Bolouri, 2008) (see Figure 4). An alternative signalling mechanism is via morphogens - a class of signalling molecule capable of providing cells with positional information. Emitted by a source, morphogens are diffusible molecules that set up a gradient to which cells then exhibit a concentration-dependent response (Wolpert, 1969; Mjolsness et al., 1991; Meinhardt and Gierer, 2000).

Theoretical studies using ensembles have compared the types of patterns exhibited by GRNs where interactions between cells occurred via either hierarchical or reciprocal fashion (SalazarCiudad et al., 2000). A key finding of this research was that, in the same way that ordered 
behaviours such as attractors can be robustly obtained from arbitrary networks satisfying certain topological constraints, so too can ordered spatial patterns. Furthermore, complex spatial patterns could be reduced to a combination of simpler modules, suggesting that, like control motifs in networks, evolution has assembled complex phenotypes from simpler building blocks.

During development, cells not only change their internal state and interact chemically, they also change their physical form and interact in a mechanical fashion. These morphogenetic mechanisms include differential growth rates across a cellular field, cell migration, apoptosis and differential cell adhesion (Salazar-Ciudad et al., 2003). These physical changes to the conformation of cells can influence a GRN's dynamics by changing its environment, and the external signals it receives, over the course of development. An accurate depiction of morphogenesis can demand more complex models in which a GRN is supplemented by a morphogenetic model. The construction of simulation environments that reflect the complexity of development whilst remaining computationally tractable is an area of ongoing research (Cickovski et al., 2005; Merks et al., 2006).

\section{Conclusion}

Understanding the dynamic behaviour of GRNs is central to our understanding of development and of developmental diseases. Dynamical systems models are important tools for this task, driving both the discovery of new theoretical insights and the integration of new sources

of empirical data. Models of GRNs assist us both to understand the intricate patterns of interaction within a regulatory system, and to investigate the system's response to internal and external perturbations. The robustness of this response informs us about the tolerances that a system has evolved, and the failure of this robustness can be viewed as a precursor to systemic diseases such as cancer (Kitano, 2003, 2004), as well as abnormal developmental pathways (Albert and Othmer, 2003).

Current challenges in dynamical models arise from a number of sources. New modelling methodologies are needed to facilitate the construction of more computationally efficient models that are able to cope with the realities of stochasticity and morphological complexity on a larger and more detailed scale. Many questions still remain about the trade-offs between reliability and efficiency inherent in robust systems. Similarly, elucidating the role that modularity plays in the organisation of GRNs remains an open question. Perhaps the most challenging avenue, and one offering some of the most promising developments, is the further integration of theoretical models with real systems. The arrival of new types of experimental data and the discovery of new regulatory mechanisms poses a challenge for theoreticians to integrate this 
knowledge into their models. In exchange, the development of more sophisticated techniques for the analysis of network dynamics will provide empiricists with better tools for interpreting the behaviour of the systems they study.

\section{Acknowledgements}

The first author acknowledges financial support by the EPSRC (grant ref: EP/D00232X/1). The second author acknowledges financial support by the European Union through the project MEXT-CT-2006-042484 ("PLICON"), and by the Hertie Foundation.

\section{References}

Albert, R. (2005). Scale-free networks in cell biology. J Cell Sci, 118(21):4947-4957.

Albert, R. and Barabási, A.-L. (2002). Statistical mechanics of complex networks. Rev Mod Phys, 74:47-97.

Albert, R., Jeong, H., and Barabasi, A.-L. (2000). Error and attack tolerance of complex networks. Nature, 406:378-82.

Albert, R. and Othmer, H. G. (2003). The topology of the regulatory interactions predicts the expression pattern of the segment polarity genes in Drosophila melanogaster. $J$ Theor Biol, $223: 1-18$.

Aldana, M. (2003). Boolean dynamics of networks with scale-free topology. Phys Nonlinear Phenom, 185:45-66.

Aldana, M., Balleza, E., Kauffman, S. A., and Resendiz, O. (2007). Robustness and evolvability in genetic regulatory networks. J Theor Biol, 245:433-448.

Alon, U. (2007). Network motifs: theory and experimental approaches. Nat Rev Genet, 8:450461.

Angeli, D., Ferrell, J. E., and Sontag, E. D. (2004). Detection of multistability, bifurcations, and hysteresis in a large class of biological positive-feedback systems. Proc Natl Acad Sci USA, 101:1822-1827.

Balleza, E., Alvarez-Buylla, E. R., Chaos, Á., Kauffman, S. A., Shmulevich, I., and Aldana, M. (2008). Critical dynamics in genetic regulatory networks: examples from four kingdoms. PLoS One, 3:e2456.

Barabási, A.-L. and Albert, R. (1999). Emergence of scaling in random networks. Science, 286:509-512.

Ben-Tabou de-Leon, S. and Davidson, E. H. (2007). Gene regulation: gene control network in development. Annu Rev Biophys Biomol Struct, 36:191-212. 
Blake, W. J., Kærn, M., Cantor, C. R., and Collins, J. J. (2003). Noise in eukaryotic gene expression. Nature, 422:633-637.

Bolouri, H. (2008). Embryonic pattern formation without morphogens. BioEssays, 30:412-417.

Bolouri, H. and Davidson, E. H. (2002). Modeling transcriptional regulatory networks. BioEssays, 24:1118-1129.

Braunewell, S. and Bornholdt, S. (2007). Superstability of the yeast cell-cycle dynamics: ensuring causality in the presence of biochemical stochasticity. $J$ Theor Biol, 245:638-643.

Brazma, A. and Schlitt, T. (2003). Reverse engineering of gene regulatory networks: a finite state linear model. Genome Biol, 4:P5.

Carroll, S. B., Grenier, J. K., and Weatherbee, S. D. (2001). From DNA to Diversity: Molecular Genetics and the Evolution of Animal Design. Blackwell Science, Oxford.

Chan, H. Y. E. and Bonini, N. M. (2000). Drosophila models of human neurodegenerative disease. Cell Death Differ, 7:1075-1080.

Chaos, Á., Aldana, M., Espinosa-Soto, C., de León, B., Arroyo, A., and Alvarez-Buylla, E. (2006). From genes to flower patterns and evolution: dynamic models of gene regulatory networks. J Plant Growth Regul, 25:278-289.

Chaves, M., Albert, R., and Sontag, E. D. (2005). Robustness and fragility of Boolean models for genetic regulatory networks. J Theor Biol, 235:431-449.

Chaves, M., Sontag, E. D., and Albert, R. (2006). Methods of robustness analysis for Boolean models of gene control networks. Syst Biol, 153:154-167.

Chen, T., He, H. L., and Church, G. M. (1999). Modeling gene expression with differential equations. In Altman, R. B., Lauderdale, K., Dunker, A. K., Hunter, L., and Klein, T. E., editors, Pacific Symposium on Biocomputing '99, Singapore. World Scientific.

Cickovski, T. M., Huang, C., Chaturvedi, R., Glimm, T., Hentschel, H. G. E., Alber, M. S., Glazier, J. A., Newman, S. A., and Izaguirre, J. A. (2005). A framework for threedimensional simulation of morphogenesis. IEEE ACM Trans Comput Biol Bioinformatics, $2: 273-288$.

Davidich, M. I. and Bornholdt, S. (2008a). Boolean network model predicts cell cycle sequence of fission yeast. PLoS One, 3:e1672.

Davidich, M. I. and Bornholdt, S. (2008b). The transition from differential equations to Boolean networks: a case study in simplifying a regulatory network model. J Theor Biol, 255:269-277.

Davidson, E. H. (2001). Genomic Regulatory Systems. Academic Press, San Diego, CA.

Davidson, E. H. and Levine, M. S. (2008). Properties of developmental gene regulatory networks. Proc Natl Acad Sci USA, 105:20063-20066.

Davidson, E. H., Rast, J. P., Oliveri, P., Ransick, A., Calestani, C., Yuh, C., Minokawa, T., Amore, G., Hinman, V., Arenas-Mena, C., Otim, O., Brown, C. T., Livi, C. B., Lee, P. Y., 
Revilla, R., Rust, A. G., jun Pan, Z., Schilstra, M. J., Clarke, P. J. C., Arnone, M. I., Rowen, L., Cameron, R. A., McClay, D. R., Hood, L., and Bolouri, H. (2002). A genomic regulatory network for development. Science, 295:1669-1678.

de Jong, H., Geiselmann, J., Batt, G., Hernandez, C., and Page, M. (2004a). Qualitative simulation of the initiation of sporulation in Bacillus subtilis. Bull Math Biol, 66:261-299.

de Jong, H., Gouzé, J., Hernandez, C., Page, M., Sari, T., and Geiselmann, J. (2004b). Qualitative simulation of genetic regulatory networks using piecewise-linear models. Bull Math Biol, 66:301-340.

Derrida, B. and Pomeau, Y. (1986). Random networks of automata: a simple annealed approximation. Europhys Lett, 1:45-49.

D'haeseleer, P., Wen, X., Fuhrman, S., and Somogyi, R. (1999). Linear modeling of mRNA expression levels during CNS development and injury. In Altman, R. B., Lauderdale, K., Dunker, A. K., Hunter, L., and Klein, T. E., editors, Pacific Symposium on Biocomputing '99, pages 41-52. World Scientific, Singapore.

Di Paolo, E. A. (2001). Rhythmic and non-rhythmic attractors in asynchronous random Boolean networks. BioSystems, 59:185-195.

Edwards, R. and Glass, L. (2000). Combinatorial explosion in model gene networks. Chaos, 10:691-704.

Edwards, T. M. and Myers, J. P. (2007). Environmental exposures and gene regulation in disease etiology. Environ Health Perspect, 115:1264-1270.

Espinosa-Soto, C., Padilla-Longoria, P., and Alvarez-Buylla, E. R. (2004). A gene regulatory network model for cell-fate determination during Arabidopsis thaliana flower development that is robust and recovers experimental gene expression profiles. Plant Cell, 16:2923-2939.

Forger, D. B. and Peskin, C. S. (2005). Stochastic simulation of the mammalian circadian clock. Proc Natl Acad Sci USA, 102:321-324.

Gillespie, D. T. (1977). Exact stochastic simulation of coupled chemical reactions. J Phys Chem, 81:2340-2361.

Gillespie, D. T. (2001). Approximate accelerated stochastic simulation of chemically reacting systems. J Chem Phys, 115:1716-1733.

Goutsias, J. (2007). Classical versus stochastic kinetics modeling of biochemical reaction systems. Biophys J, 92:2350-2365.

Goutsias, J. and Kim, S. (2004). A nonlinear discrete dynamical model for transcriptional regulation: construction and properties. Biophys J, 86:1922-1945.

Harris, S. E., Sawhill, B. K., Wuensche, A., and Kauffman, S. A. (2002). A model of transcriptional regulatory networks based on biases in the observed regulation rules. Complexity, $7(4): 23-40$.

Hasty, J., McMillen, D., Isaacs, F., and Collins, J. J. (2001). Computational studies of gene 
regulatory networks: in numero molecular biology. Nat Rev Genet, 2:268-279.

Hertz, J., Krogh, A., and Palmer, R. G. (1991). Introduction to the Theory of Neural Computation. Addison Wesley, Redwood City, CA.

Huang, S. (2004). Regulation of cellular states in mammalian cells from a genomewide view. In Collado-Vides, J. and Hofestädt, R., editors, Gene Regulation and Metabolism: Postgenomic Computational Approaches, pages 181-220. MIT Press, Cambridge, MA.

Huang, S., Eichler, G., Bar-Yam, Y., and Ingber, D. E. (2005). Cell fates as high-dimensional attractor states of a complex gene regulatory network. Phys Rev Lett, 94:128701.

Huang, S., Guo, Y., May, G., and Enver, T. (2007). Bifurcation dynamics in lineagecommitment in bipotent progenitor cells. Dev Biol, 305:695-713.

Hucka, M., Finney, A., Sauro, H. M., Bolouri, H., Doyle, J. C., Kitano, H., Arkin, A. P., Bornstein, B. J., Bray, D., Cornish-Bowden, A., Cuellar, A. A., Dronov, S., Gilles, E. D., Ginkel, M., Gor, V., Goryanin, I. I., Hedley, W. J., Hodgman, T. C., Hofmeyr, J., Hunter, P. J., Juty, N. S., Kasberger, J. L., Kremling, A., Kummer, U., Novère, N. L., Loew, L. M., Lucio, D., Mendes, P., Minch, E., Mjolsness, E. D., Nakayama, Y., Nelson, M. R., Nielsen, P. F., Sakurada, T., Schaff, J. C., Shapiro, B. E., Shimizu, T. S., Spence, H. D., Stelling, J., Takahashi, K., Tomita, M., Wagner, J., and Wang, J. (2003). The systems biology markup language (SBML): a medium for representation and exchange of biochemical network models. Bioinformatics, 19:524-31.

Irons, D. and Monk, N. (2007). Identifying dynamical modules from genetic regulatory systems: applications to the segment polarity network. BMC Bioinformatics, 8:413.

Johnston, R. J., Chang, S., Etchberger, J. F., Ortiz, C. O., and Hobert, O. (2005). MicroRNAs acting in a double-negative feedback loop to control a neuronal cell fate decision. Proc Natl Acad Sci USA, 102:12449-12454.

Kauffman, S. A. (1969). Metabolic stability and epigenesis in randomly constructed genetic nets. J Theor Biol, 22:437-467.

Kauffman, S. A. (1971). Gene regulation networks: a theory for their global structure and behaviours. Curr Top Dev Biol, 6:145-182.

Kauffman, S. A. (1993). The Origins of Order: self-organization and selection in evolution. Oxford University Press, Oxford.

Kauffman, S. A. (2004a). The ensemble approach to understand genetic regulatory networks. Phys Stat Mech Appl, 340:733-740.

Kauffman, S. A. (2004b). A proposal for using the ensemble approach to understand genetic regulatory networks. J Theor Biol, 231:581-590.

Kauffman, S. A., Peterson, C., Samuelsson, B., and Troein, C. (2003). Random Boolean network models and the yeast transcriptional network. Proc Natl Acad Sci USA, 100:1479614799. 
Kauffman, S. A., Peterson, C., Samuelsson, B., and Troein, C. (2004). Genetic networks with canalyzing Boolean rules are always stable. Proc Natl Acad Sci USA, 101:17102-17107.

Kim, J., Kim, T., Jung, S. H., Kim, J., Park, T., Heslop-Harrison, P., and Cho, K. (2008). Evolutionary design principles of modules that control cellular differentiation: consequences for hysteresis and multistationarity. Bioinformatics, 24:1516-1522.

Kitano, H. (2001). Foundations of Systems Biology. The MIT Press/Bradford Books, Cambridge, MA.

Kitano, H. (2002). Systems biology: a brief overview. Science, 295:1662-1664.

Kitano, H. (2003). Cancer robustness: tumour tactics. Nature, 426(6963):125.

Kitano, H. (2004). Biological robustness. Nat Rev Genet, 5:826-837.

Klemm, K. and Bornholdt, S. (2005). Topology of biological networks and reliability of information processing. Proc Natl Acad Sci USA, 102:18414-18419.

Kouretas, P., Koutroumpas, K., Lygeros, J., and Lygerou, Z. (2006). Stochastic hybrid modeling of biochemical processes. In Cassandras, C. and Lygeros, J., editors, Stochastic Hybrid Systems, pages 221-248. CRC Press, Boca Raton, FL.

Levine, M. and Davidson, E. H. (2005). Gene regulatory networks for development. Proc Natl Acad Sci USA, 102:4936-4942.

Li, F., Long, T., Lu, Y., Ouyang, Q., and Tang, C. (2004). The yeast cell-cycle network is robustly designed. Proc Natl Acad Sci USA, 101:4781-4786.

Longabaugh, W. J., Davidson, E. H., and Bolouri, H. (2008). Visualization, documentation, analysis, and communication of large-scale gene regulatory networks. Biochim Biophys Acta, In Press, Corrected Proof.

Losick, R. and Desplan, C. (2008). Stochasticity and cell fate. Science, 320:65-68.

Luque, B. and Solé, R. V. (2000). Lyapunov exponents in random Boolean networks. Phys Stat Mech Appl, 284:33-45.

Mahaffy, J. M. (1988). Genetic control models with diffusion and delays. Math Biosci, 90:519533.

Mahaffy, J. M. and Pao, C. V. (1984). Models of genetic control by repression with time delays and spatial effects. J Math Biol, 20:39-57.

McAdams, H. H. and Arkin, A. (1997). Stochastic mechanisms in gene expression. Proc Natl Acad Sci USA, 94:814-819.

McAdams, H. H. and Shapiro, L. (1995). Circuit simulation of genetic networks. Science, 269:650-656.

Meinhardt, H. and Gierer, A. (2000). Pattern formation by local self-activation and lateral inhibition. BioEssays, 22:753-760.

Meir, E., von Dassow, G., Munro, E., and Odell, G. M. (2002). Robustness, flexibility, and the role of lateral inhibition in the neurogenic network. Curr Biol, 12:778-786. 
Mendoza, L. and Alvarez-Buylla, E. R. (1998). Dynamics of the genetic regulatory network for Arabidopsis thaliana flower morphogenesis. J Theor Biol, 193:307-19.

Merks, R. M. H., Hoekstra, A. G., Kaandorp, J. A., Sloot, P. M. A., and Hogeweg, P. (2006). Problem-solving environments for biological morphogenesis. Comp Sci Eng, 8:61-72.

Mestl, T., Plahte, E., and Omholt, S. W. (1995). A mathematical framework for describing and analysing gene regulatory networks. J Theor Biol, 176:291-300.

Mjolsness, E., Sharp, D. H., and Reinitz, J. (1991). A connectionist model of development. J Theor Biol, 152:429-53.

Monk, N. A. M. (2003). Oscillatory expression of hes1, p53, and NF-kappaB driven by transcriptional time delays. Current Biology: CB, 13:1409-13.

Novak, B. and Tyson, J. (1993). Numerical analysis of a comprehensive model of m-phase control in Xenopus oocyte extracts and intact embryos. J Cell Sci, 106:1153-1168.

Oliveri, P., Tu, Q., and Davidson, E. H. (2008). Global regulatory logic for specification of an embryonic cell lineage. Proc Natl Acad Sci USA, 105:5955-5962.

Olson, E. N. (2006). Gene regulatory networks in the evolution and development of the heart. Science, 313:1922-1927.

Orphanides, G. and Reinberg, D. (2002). A unified theory of gene expression. Cell, 108:439451.

Osada, R., Zaslavsky, E., and Singh, M. (2004). Comparative analysis of methods for representing and searching for transcription factor binding sites. Bioinformatics, 20:3516-25.

Quackenbush, J. (2007). Extracting biology from high-dimensional biological data. J Exp Biol, 210:1507-1517.

Rao, C. V., Wolf, D. M., and Arkin, A. P. (2002). Control, exploitation and tolerance of intracellular noise. Nature, 420:231-237.

Raser, J. M. and O'Shea, E. K. (2004). Control of stochasticity in eukaryotic gene expression. Science, 304:1811-1814.

Rohlfshagen, P. and Di Paolo, E. A. (2004). The circular topology of rhythm in asynchronous random Boolean networks. BioSystems, 73:141-152.

Salazar-Ciudad, I., Garcia-Fernández, J., and Solé, R. V. (2000). Gene networks capable of pattern formation: from induction to reaction-diffusion. J Theor Biol, 205:587-603.

Salazar-Ciudad, I., Jernvall, J., and Newman, S. A. (2003). Mechanisms of pattern formation in development and evolution. Development, 130:2027-2037.

Samuelsson, B. and Troein, C. (2003). Superpolynomial growth in the number of attractors in Kauffman networks. Phys Rev Lett, 90(9):098701.

Savageau, M. A. (2001). Design principles for elementary gene circuits: elements, methods, and examples. Chaos, 11:142-159.

Schadt, E. E. and Lum, P. Y. (2006). Systems biology approaches to metabolic and cardiovas- 
cular disorders. reverse engineering gene networks to identify key drivers of complex disease phenotypes. J Lipid Res, 47:2601-2613.

Schlitt, T. and Brazma, A. (2007). Current approaches to gene regulatory network modelling. BMC Bioinformatics, 8:S9.

Shannon, P., Markiel, A., Ozier, O., Baliga, N. S., Wang, J. T., Ramage, D., Amin, N., Schwikowski, B., and Ideker, T. (2003). Cytoscape: a software environment for integrated models of biomolecular interaction networks. Genome Res, 13:2498-504.

Shea, M. A. and Ackers, G. K. (1985). The OR control system of bacteriophage lambda. a physical-chemical model for gene regulation. J Mol Biol, 181:211-230.

Shmulevich, I., Kauffman, S. A., and Aldana, M. (2005). Eukaryotic cells are dynamically ordered or critical but not chaotic. Proc Natl Acad Sci USA, 102(38):13439-13444.

Smith, J., Theodoris, C., and Davidson, E. H. (2007). A gene regulatory network subcircuit drives a dynamic pattern of gene expression. Science, 318:794-797.

Smolen, P., Baxter, D. A., and Byrne, J. H. (1999). Effects of macromolecular transport and stochastic fluctuations on dynamics of genetic regulatory systems. Am J Physiol, 277(4 Pt 1):C777-C790.

Socolar, J. E. S. and Kauffman, S. A. (2003). Scaling in ordered and critical random Boolean networks. Phys Rev Lett, 90(6):068702.

Strogatz, S. (1994). Nonlinear Dynamics and Chaos. Addison-Wesley, Reading, MA.

Süel, G. M., Kulkarni, R. P., Dworkin, J., Garcia-Ojalvo, J., and Elowitz, M. B. (2007). Tunability and noise dependence in differentiation dynamics. Science, 315:1716-1719.

Swain, P. S., Elowitz, M. B., and Siggia, E. D. (2002). Intrinsic and extrinsic contributions to stochasticity in gene expression. Proc Natl Acad Sci USA, 99:12795-12800.

Swiers, G., Patient, R., and Loose, M. (2006). Genetic regulatory networks programming hematopoietic stem cells and erythroid lineage specification. Dev Biol, 294:525-540.

Thattai, M. and van Oudenaarden, A. (2001). Intrinsic noise in gene regulatory networks. Proc Natl Acad Sci USA, 98:8614-8619.

Thieffry, D. and Thomas, R. (1995). Dynamical behaviour of biological regulatory networks-II: immunity control in bacteriophage lambda. Bull Math Biol, 57:277-297.

Thomas, R. (1973). Boolean formalization of genetic control circuits. J Theor Biol, 42:563-585.

Thomas, R. (1998). Laws for the dynamics of regulatory networks. Int J Dev Biol, 42:479-485.

Thomas, R. and Kaufman, M. (1995). Dynamical behaviour of biological regulatory networksI: biological role of feedback loops and practical use of the concept of the loop-characteristic state. Bull Math Biol, 57:247-276.

Tyson, J. J., Chen, K., and Novak, B. (2001). Network dynamics and cell physiology. Nat Rev Mol Cell Biol, 2:908-916.

Tyson, J. J., Chen, K. C., and Novak, B. (2003). Sniffers, buzzers, toggles and blinkers: 
dynamics of regulatory and signaling pathways in the cell. Curr Opin Cell Biol, 15:221-231.

Tyson, J. J., Csikasz-Nagy, A., and Novak, B. (2002). The dynamics of cell cycle regulation. BioEssays, 24:1095-1109.

Vohradský, J. (2001). Neural network model of gene expression. Faseb J, 15:846-854.

von Dassow, G., Meir, E., Munro, E. M., and Odell, G. M. (2000). The segment polarity network is a robust developmental module. Nature, 406:188-192.

von Dassow, G. and Odell, G. M. (2002). Design and constraints of the Drosophila segment polarity module: robust spatial patterning emerges from intertwined cell state switches. $J$ Exp Zool, 294:179-215.

Wahde, M. and Hertz, J. (2001). Modeling genetic regulatory dynamics in neural development. J Comput Biol, 8:429-42.

Watts, D. J. and Strogatz, S. H. (1998). Collective dynamics of 'small-world' networks. Nature, 393:440-442.

Weaver, D. C., Workman, C. T., and Stormo, G. D. (1999). Modeling regulatory networks with weight matrices. In Altman, R. B., Lauderdale, K., Dunker, A. K., Hunter, L., and Klein, T. E., editors, Pacific Symposium on Biocomputing '99, pages 112-123, Singapore. World Scientific.

Willadsen, K., Triesch, J., and Wiles, J. (2008). Understanding robustness in random Boolean networks. In Bullock, S., Noble, J., Watson, R., and Bedau, M. A., editors, Artificial Life XI: Proceedings of the Eleventh International Conference on the Simulation and Synthesis of Living Systems, pages 694-701. MIT Press, Cambridge, MA.

Willadsen, K. and Wiles, J. (2007). Robustness and state-space structure of Boolean gene regulatory models. J Theor Biol, 249(4):749-765.

Wolpert, L. (1969). Positional information and the spatial pattern of cellular differentiation. $J$ Theor Biol, 25:1-47.

Xiong, W. and Ferrell, J. E. (2003). A positive-feedback-based bistable 'memory module' that governs a cell fate decision. Nature, 426:460-465.

Yuh, C., Bolouri, H., and Davidson, E. H. (1998). Genomic cis-regulatory logic: experimental and computational analysis of a sea urchin gene. Science, 279:1896-1902.

Zhu, R., Ribeiro, A. S., Salahub, D., and Kauffman, S. A. (2007). Studying genetic regulatory networks at the molecular level: delayed reaction stochastic models. J Theor Biol, 246:725745 . 


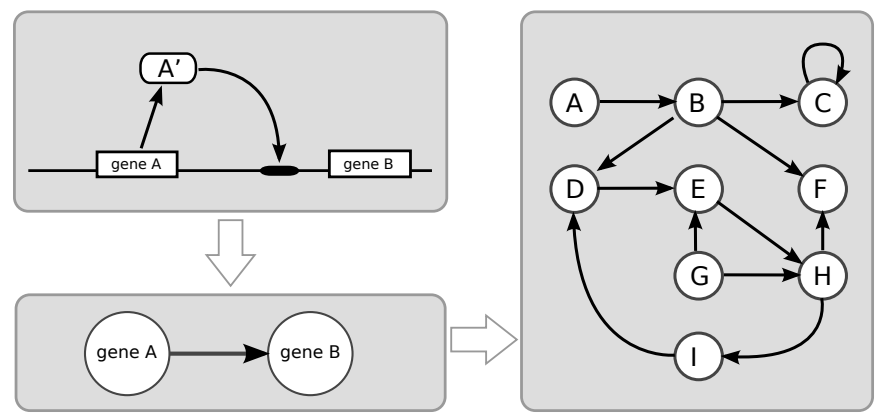

Figure 1: A typical figurative representation of a gene interaction-gene $A$ produces product $A^{\prime}$, which exerts a regulatory influence on gene $B$ (top left), its network-based analogue (bottom left) and a whole regulatory network (right).
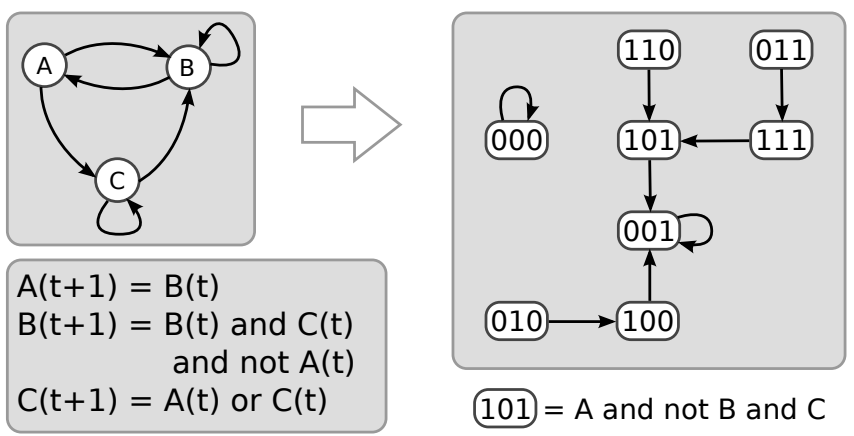

Figure 2: A three-node RBN with the logical updating functions of its nodes (left) and the corresponding state space (right). Nodes in the state space with self-loops are the network's attractors; connected sets of nodes are basins of attraction. 

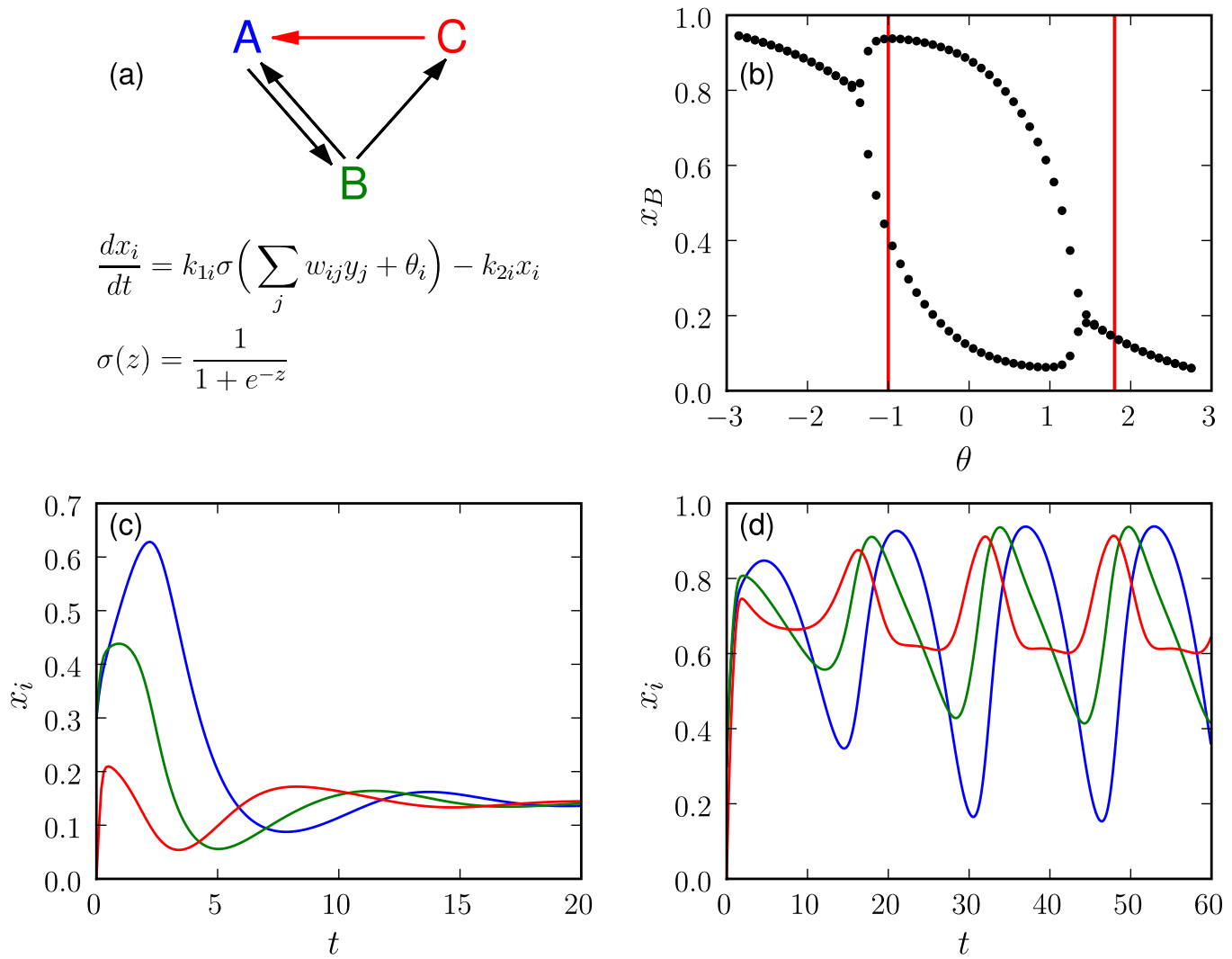

Figure 3: A DE model of a simple three-node network. (a) The network wiring diagram and update functions. Black/red arrows indicate activatory/inhibitory regulation. The update equation describes how $x_{i}$, the expression level of gene $i$, changes over time, where: $w_{i j}$ represents the level of influence that gene $j$ has on gene $i$; $\theta_{i}$ represents the basal level of expression of gene $i$, and $k_{1 i}$ and $k_{2 i}$ are constants representing, respectively, the maximum expression level and degradation rate of gene $i . \sigma$ is the logistic sigmoid transfer function. (b) A bifurcation diagram showing the ranges of $\theta$ for which the network exhibits fixed point and periodic behaviour; The minimum and maximum values of $x_{B}$ for each value of $\theta$ are plotted on the vertical axis. The two red bars indicate the time courses shown in plots c and d. (c) $\theta=1.8$ : After a transient period, the network settles to a stable fixed point. (d) $\theta=-0.8$ : After a transient period, the network settles to a stable periodic oscillation. Parameter values for all simulations: $w_{A C}=-10.0 ; w_{A B}=w_{B A}=w_{C B}=10.0 ; w_{C A}=$ $w_{C B}=w_{A A}=w_{B B}=w_{C C}=0.0 ; k_{1}=k_{2}=1.0$ for all $i$. 


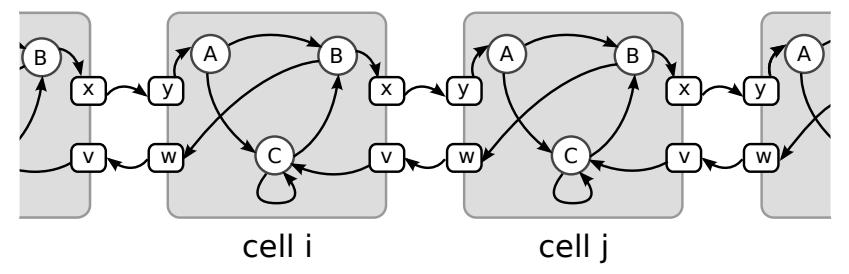

Figure 4: A GRN embedded in one-dimensional cellular space. This wiring diagram illustrates cellular communication taking place by way of inductive signals between neighbouring cells. 